\title{
Breast cancer cells obtain an osteomimetic feature via epithelial- mesenchymal transition that have undergone BMP2/RUNX2 signaling pathway induction
}

\author{
Cong-Cong Tan ${ }^{1, *}$, Gui-Xi Li ${ }^{1, *}$, Li-Duan Tan ${ }^{1}$, Xin Du ${ }^{1}$, Xiao-Qing Li ${ }^{1,2}$, Rui He ${ }^{1}$, Qing- \\ Shan Wang ${ }^{1,2}$, Yu-Mei Feng ${ }^{1,2}$ \\ ${ }^{1}$ Department of Biochemistry and Molecular Biology, Tianjin Medical University Cancer Institute and Hospital, National Clinical \\ Research Center of Cancer, Tianjin 300060, China \\ ${ }^{2}$ Key Laboratory of Breast Cancer Prevention and Treatment of the Ministry of Education, Tianjin Medical University Cancer \\ Institute and Hospital, National Clinical Research Center of Cancer, Tianjin 300060, China \\ *These authors have contributed equally to this work \\ Correspondence to: Yu-Mei Feng, email: ymfeng@tmu.edu.cn \\ Keywords: osteomimicry, epithelial-mesenchymal transition, BMP2/RUNX2 signaling pathway, breast cancer bone metastasis, \\ cancer-associated fibroblast \\ Received: March 24, $2016 \quad$ Accepted: September 25, $2016 \quad$ Published: October 27, 2016
}

\section{ABSTRACT}

Bone is one of the most common organs of breast cancer metastasis. Cancer cells that mimic osteoblasts by expressing bone matrix proteins and factors have a higher likelihood of metastasizing to bone. However, the molecular mechanisms of osteomimicry formation of cancer cells remain undefined. Herein, we identified a set of bone-related genes (BRGs) that are ectopically co-expressed in primary breast cancer tissues and determined that osteomimetic feature is obtained due to the osteoblast-like transformation of epithelial breast cancer cells that have undergone epithelial-mesenchymal transition (EMT) followed by bone morphogenetic protein-2 (BMP2) stimulation. Furthermore, we demonstrated that breast cancer cells that transformed into osteoblast-like cells with high expression of BRGs showed enhanced chemotaxis, adhesion, proliferation and multidrug resistance in an osteoblast-mimic bone microenvironment in vitro. During these processes, runt-related transcription factor 2 (RUNX2) functioned as a master mediator by suppressing or activating the transcription of BRGs that underlie the dynamic antagonism between the TGF- $\beta$ / SMAD and BMP/SMAD signaling pathways in breast cancer cells. Our findings suggest a novel mechanism of osteomimicry formation that arises in primary breast tumors, which may explain the propensity of breast cancer to metastasize to the skeleton and contribute to potential strategies for predicting and targeting breast cancer bone metastasis and multidrug resistance.

\section{INTRODUCTION}

Breast cancer commonly metastasizes to the bone in women with advanced disease, which can cause debilitating skeletal complications, such as bone destruction and associated bone pain, fracture, hypercalcemia, and paralysis due to spinal cord compression [1]. The affinity of cancer cell metastases for bone relies on intrinsic biological properties [2] and specific interactions with the bone microenvironment [3]. Breast cancer cells can acquire an osteoblast-like phenotype by ectopically expressing bone matrix proteins (e.g., bone sialoprotein (BSP) [4], osteopontin (OPN) [5], osteoprotegerin (OPG) [6] and secreted protein acidic and cysteine rich (SPARC)/osteonectin (ON) [7]), osteoblastspecific cadherins (e.g., cadherin 11 (CDH11) [8]), transcription factors that regulate bone remodeling (e.g., runt-related transcription factor 2 (RUNX2) [9]) and an "osteoblast gene signature" [2]. Cancer cells that highly express these bone-related genes (BRGs) preferentially home to [6], colonize in [8, 10], and survive in [11] bone. However, the clinical relevance of BRGs to bone-specific metastasis and how osteomimetic properties emerge in primary tumors remain poorly understood. 
Cancer-associated fibroblasts (CAFs), which are activated by tumor cells, are the predominant type of stromal cell in breast cancer tissues. CAFs reciprocally promote aggressive phenotypes of breast cancer cells through paracrine signals (e.g., TGF- $\beta$ ) that trigger epithelial-to-mesenchymal transition (EMT) [12]. EMT is an important mechanism that enables cancer cells to complete various steps in the metastatic cascade by transiting to a mesenchymal cell phenotype [13] with stem cell-like properties [14]. Epithelial cancer cells that have undergone EMT exhibit multi-lineage differentiation potential similar to mesenchymal stem cells and can differentiate into myofibroblasts/CAFs [15], osteoblasts, chondrocytes and adipocytes [16] in response to tissuespecific differentiation signals. Bone morphogenetic proteins (BMPs) are key osteogenic factors that induce the maturation of mesenchymal cells into osteoblasts by activating various osteogenesis-related transcription factors, such as RUNX2 [17]. BMP2 has been reported to be expressed in breast cancer cell lines [18] and primary breast cancer tissues [19], and it is particularly elevated in breast cancer bone metastatic samples relative to metastases in other organs [20]. These pieces of evidence led us to hypothesize that the osteomimetic phenotype of breast cancer cells that ectopically coexpress BRGs may be derived from EMT followed by the induction of osteogenic differentiation signals in the tumor microenvironment, which mimics the process of osteoblastic differentiation under normal physiological conditions.

In the current study, we provided clinical evidence to reveal that breast cancers present an osteomimic feature with the ectopic co-expression of a set of BRGs which preferentially metastasize to bone. Mechanistically, we demonstrated that the ectopic co-expression of BRGs in breast cancer cells is derived from EMT that has undergone BMP2 induction. Functionally, we validated the propensity of osteomimetic breast cancer cells for chemotaxis, adhesion, proliferation and multidrug resistance in an osteoblast-mimic bone microenvironment in vitro. Furthermore, we demonstrated that RUNX2 serves as a master mediator of the formation of an osteomimetic phenotype by activating the transcription of BRGs in breast cancer cells.

\section{RESULTS}

\section{Breast cancers present an osteomimetic feature with the ectopic co-expression of a set of BRGs which has a tendency to metastasize to bone}

To investigate the intrinsic features of breast cancer, we analyzed the co-expression of genes in breast cancer tissues based on our gene expression profiling dataset of 49 primary breast cancer tissues [21] using Pearson's correlation analysis. A total of 57 BRGs were identified as co-expressed genes with a correlation coefficient greater than 0.3 in breast cancer tissues, including genes that encode bone matrix proteins (ASPN, COMP, CSPG2/VCAN, DSPG3/EPYC, POSTN, SPARC, SPOCK, COLs), extracellular matrix (ECM)-degrading enzymes (MMPs, ADAMs, ADAMTSs, CTSK, PLAU/ uPA) and their inhibitors (TIMPs), osteoblast cadherin (OB-cadherin/CDH11), osteoblast transcription factors (RUNX2), and cytokines (SCGF; Figure 1A). We then compared the mRNA levels of the set of BRGs between normal breast tissues $(n=9)$ and breast cancer tissues ( $n$ $=49$ ) based on our gene expression profiling dataset [21]. The result revealed that these BRGs were more highly expressed in breast cancer tissues than in normal breast tissues (Figure 1B and Supplementary Figures S1A-S1C). To investigate whether the ectopically co-expressed BRGs contribute to breast cancer bone metastasis, we analyzed the expression of the set of BRGs in bone metastatic breast cancer tissues $(\mathrm{n}=10)$ and metastatic breast cancer tissues in other organs $(n=19)$ based on Zhang's gene expression profiling dataset (GEO accession number: GSE14017) [20] (Figure 1C and Supplementary Figures S1D-S1F). The result showed that the set of BRGs was highly co-expressed in metastases in bone compared with those in other organs. Collectively, these findings indicate that breast cancers present an osteomimetic feature with ectopically co-expressed a set of BRGs, which have a tendency to metastasize to bone.

\section{The co-expression of BRGs in breast cancer cells is derived from EMT that has undergone BMP2 induction}

Physiologically, BRGs are initially expressed in the process of directed differentiation of bone marrow mesenchymal stem cells under the induction of growth factors during bone development and the repair of bone damage. BMP2 is a pivotal signal in the induction of bone development and bone remodeling [22] by triggering the expression of skeletal-restricted genes, such as RUNX2, $\mathrm{BSP}, \mathrm{OPN}$, and $\mathrm{OC}$, to drive the differentiation of mesenchymal cells [17]. Based on the facts that CAFs are enriched in the breast cancer microenvironment and can induce EMT in breast cancer cells [12] and that BMP2 is elevated in primary breast cancer tissues [19], we hypothesized that the ectopically co-expressed BRGs may be derived from epithelial cancer cells that have undergone EMT and acquired cancer stem cells (CSCs) properties, followed by BMP2 induction in the tumor microenvironment. To address this hypothesis, we treated epithelial breast cancer cells MCF-7 and T47D with the conditional medium from CAFs (CAF $\mathrm{CM})$ to induce EMT as previously described [12] and then added BMP2 to induce the cells that had undergone EMT to transform into an osteoblast-like phenotype. The results showed that the p-SMAD3 was dramatically 

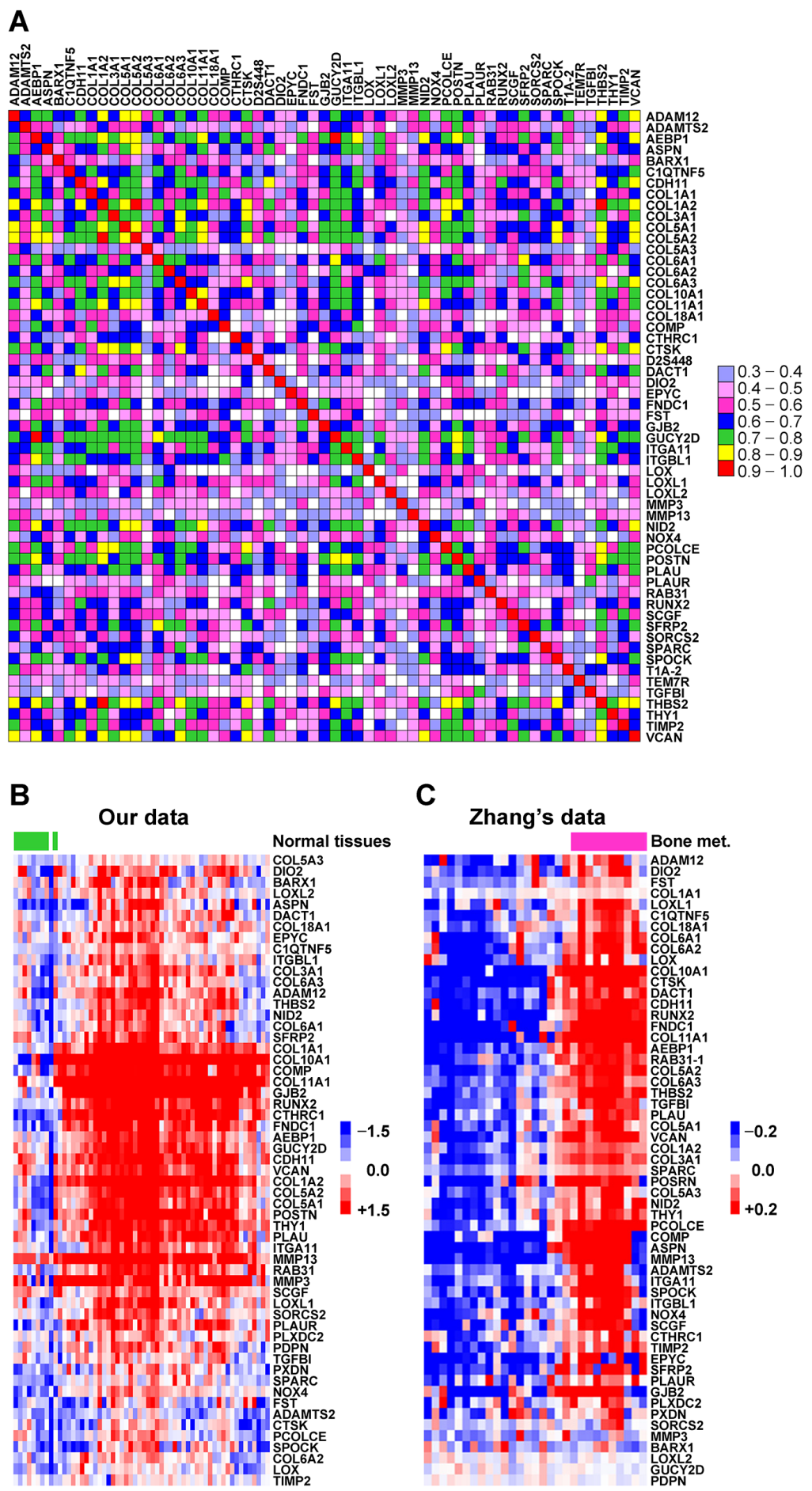

Figure 1: Breast cancers present an osteomimetic feature with the ectopic co-expression of a set of BRGs which are prone to bone metastasis. A. Schematic diagram of Pearson's correlation coefficients for the mRNA expression of 57 BRGs between each other, based on our gene expression profiling dataset of 49 primary breast cancer tissues. Correlation coefficient that greater than 0.3 is defined as a significant correlation. The color box spectrum represents different Pearson's correlation coefficients between corresponding genes. B. Comparison of mRNA expression of the set of co-expressed BRGs between normal breast tissues and breast cancer tissues. The data were mined from our gene expression profiling datasets of 9 human normal breast tissues and 49 primary breast cancer tissues. The heat map depicts the mRNA expression levels of each gene in different samples. The green bars indicate normal breast tissues, and the others are breast cancer tissues. C. Comparison of the mRNA expression of the set of co-expressed BRGs in bone metastatic breast cancer tissues and metastatic breast cancer tissues from other organ. The data were mined from Zhang's gene expression profiling dataset of 29 metastatic breast cancer tissues (GSE14017). Heat map depicts the mRNA expression levels of each gene in different samples. The purple bars indicate bone metastatic samples, and the others are metastatic samples from other organs. 
increased and p-SMAD1 was decreased by CAF CM induction, indicating the activation of the TGF- $\beta$ / SMAD signaling pathway along with the suppression of the BMP/SMAD signaling pathway. Consistently, we observed EMT phenotype in the CAF CM-treated cells: the epithelial markers E-cadherin (E-Cad) and EpCAM were downregulated, and the mesenchymal markers fibronectin 1 (FN1) and vimentin (VIM) were upregulated. When the cells that had undergone EMT in response to CAF CM were further induced by BMP2, p-SMAD1 was significantly increased and p-SMAD3 was decreased, indicating that the BMP/SMAD signaling pathway was activated along with the suppression of TGF- $\beta / \mathrm{SMAD}$ signaling pathway (Figure 2A and Supplementary Figure S2A). Consistently, we observed increased expression of the bone matrix-remodeling proteins POSTN, SPARC, CTSK, and PLAU/uPA, as well as osteoblast cadherin CDH11 and transcription factor RUNX2 (Figure 2B and Supplementary Figure S2B). However, BMP2 alone had no such effect in either MCF-7 or T47D cells (Figures 2A and $2 \mathrm{~B}$ and Supplementary Figures S2A and S2B).

To further validate the role of the EMT-BMP2 pathway in the induction of BRGs, we evaluated the expression of bone matrix-remodeling proteins in MCF7 and T47D cells treated with CAF CM followed by adding BMP2 plus noggin. The results showed that the elevated expression of RUNX2, CDH11, POSTN, SPARC, CTSK, and PLAU/uPA in CAF CM/BMP2-treated MCF7 and T47D cells was reversed by noggin (Figure 2C and Supplementary Figure S2C). Immunofluorescence staining revealed that epithelial cadherin (E-Cad) was converted into osteoblastic cadherin (CDH11) by $\mathrm{CAF} /$ BMP2 induction; however, when the BMP signaling pathway was blocked by noggin, the induction of CDH11 was attenuated (Figure 2D). These results suggest that BMP2 is essential for the induction of BRGs expression in breast cancer cells that have undergone EMT. Taken together, the above evidence demonstrates that mutual antagonism between the TGF- $\beta / \mathrm{SMAD}$ and BMP/SMAD signaling pathways regulates the transition of cells from differentiated epithelium to osteomimcry, in which the cells go through the processes of EMT and mesenchymalosteomimetic transition (MOT).

\section{Epithelial cancer cells with ectopic co-expression of BRGs inducted by CAF/BMP2 gain the advantages of homing to, residing in and growing in the bone microenvironment}

Since the osteoblast-like phenotype enables cancer cells to reside and thrive in bone [2], we further investigated whether the ectopic co-expression of BRGs in response to CAF/BMP2 induction affects the abilities of chemotaxis, adhesion, anchorage-independent growth and proliferation of epithelial cancer cells in the bone microenvironment. We used MC3T3E1 preosteoblastic cells undergone induction of osteoblast differentiation and MG-63 osteosarcoma cells or their CM to mimic the bone microenvironment in vitro to examine the chemotactic migration of MCF-7 and T47D cells towards MC3T3E1 or MG-63 cells in co-culture, the heterogeneity adhesion of cancer cells to MC3T3E1 or MG-63 cells, and the soft agar colony formation and proliferation of cancer cells in MC3T3E1 or MG-63 CM. The results showed that both MCF-7 and T47D cells cultured with CAF CM showed an EMT phenotype, with a spindle-like shape and cell scattering, while the cells changed to exhibit a polygonalshaped osteoblast-like morphology with thick pseudopodia after further BMP2 induction (Figure 3A). The chemotactic migration of MCF-7 and T47D cells towards MC3T3E1 or MG-63 cells (Figure 3B and Supplementary Figure S3A), heterogeneity adhesion to MC3T3E1 or MG-63 cells (Figure 3C and Supplementary Figure S3B), anchorage-independent growth in soft agar (Figure 3D and Supplementary Figure S3C) and proliferation (Figure 3E) in MC3T3E1 or MG-63 CM were significantly increased after CAF CM/BMP2 induction, while these enhanced capabilities were attenuated by noggin. However, BMP2 induction alone did not alter these behaviors (Figures 3A-3E and Supplementary Figures S3A-S3C). These results indicate that epithelial cancer cells with ectopic co-expression of BRGs that were derived from EMT undergone BMP2 induction acquire the capabilities to home to, reside in and grow in the bone microenvironment.

\section{RUNX2 is a critical mediator for CAF/BMP2- induced expression of BRGs in breast cancer cells}

RUNX2 is an essential transcription factor for osteoblast differentiation and bone remodeling by directly regulating multiple skeletal-restricted genes [23]. To investigate whether RUNX2 mediates the ectopic expression of $\mathrm{BRG}$ during $\mathrm{CAF} / \mathrm{BMP} 2$ induction in epithelial cancer cells, we knocked down RUNX2 expression in MCF-7 and T47D cells by RUNX2 siRNA (siRUNX2) transfection in the presence of BMP2 for 3 days after cultured with CAF CM for 3 days. We found that RUNX2 depletion resulted in significant downregulation of the $\mathrm{CAF} / \mathrm{BMP} 2$-induced expression of CDH11, POSTN, SPARC, CTSK, and PLAU/uPA compared with the control (Figure 4A and Supplementary Figure S4A). CDH11 expression was verified by immunofluorescence (Supplementary Figure S4B). Conversely, RUNX2 knockdown in MDA-MB-231 cells, a bone metastatic breast cancer cell line with high RUNX2 expression [24], down-regulated the expression of these BRGs (Figure 4A and Supplementary Figure S4A). However, the forced expression of RUNX2 in MCF-7 and T47D cells cultured with either Control CM or CAF CM did not significantly alter the protein expression levels of these BRGs, although SPARC changed a small amount in MCF-7 cells and CDH11 in T47D cells (Figure 4B and 
A

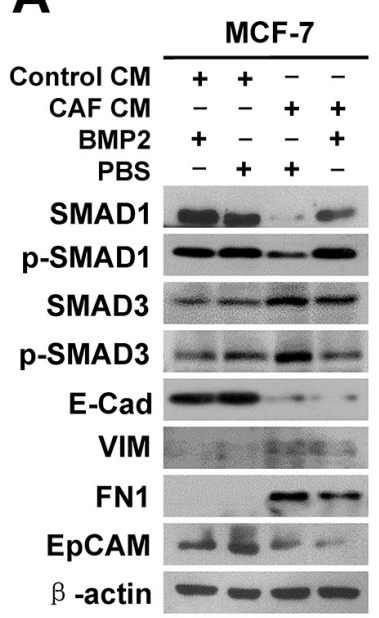

C

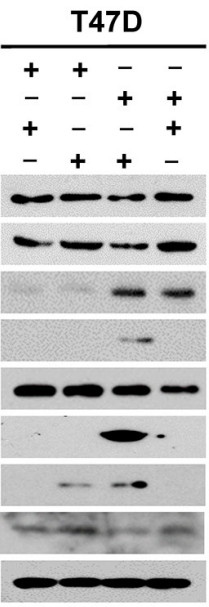

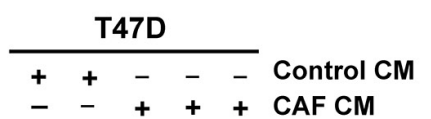

-++++ CAF CM

$+-\quad+-$ BMP2

$-\quad-\quad+$ Noggin

$-++-\quad$ PBS

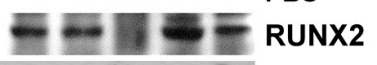

$-\longrightarrow$ CDH11

$\cdots-\cdots-$ POSTN

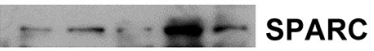

$--\infty$ CTSK

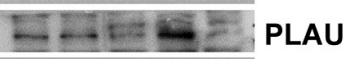

$\longrightarrow-\infty-a c t i n$
B

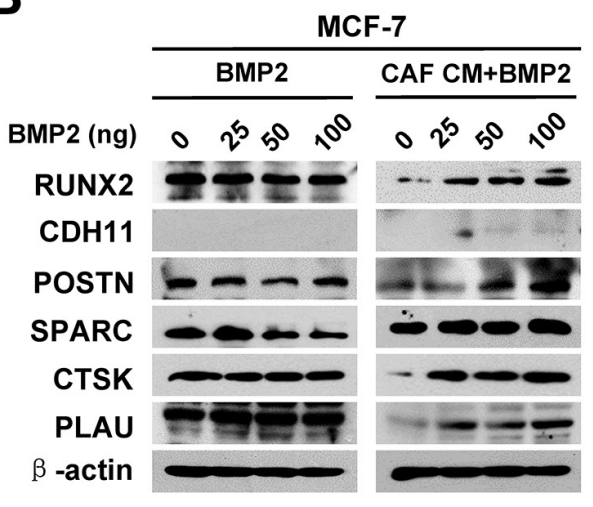

T47D

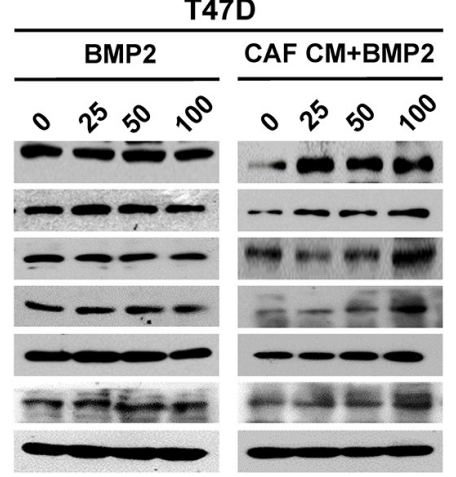

D

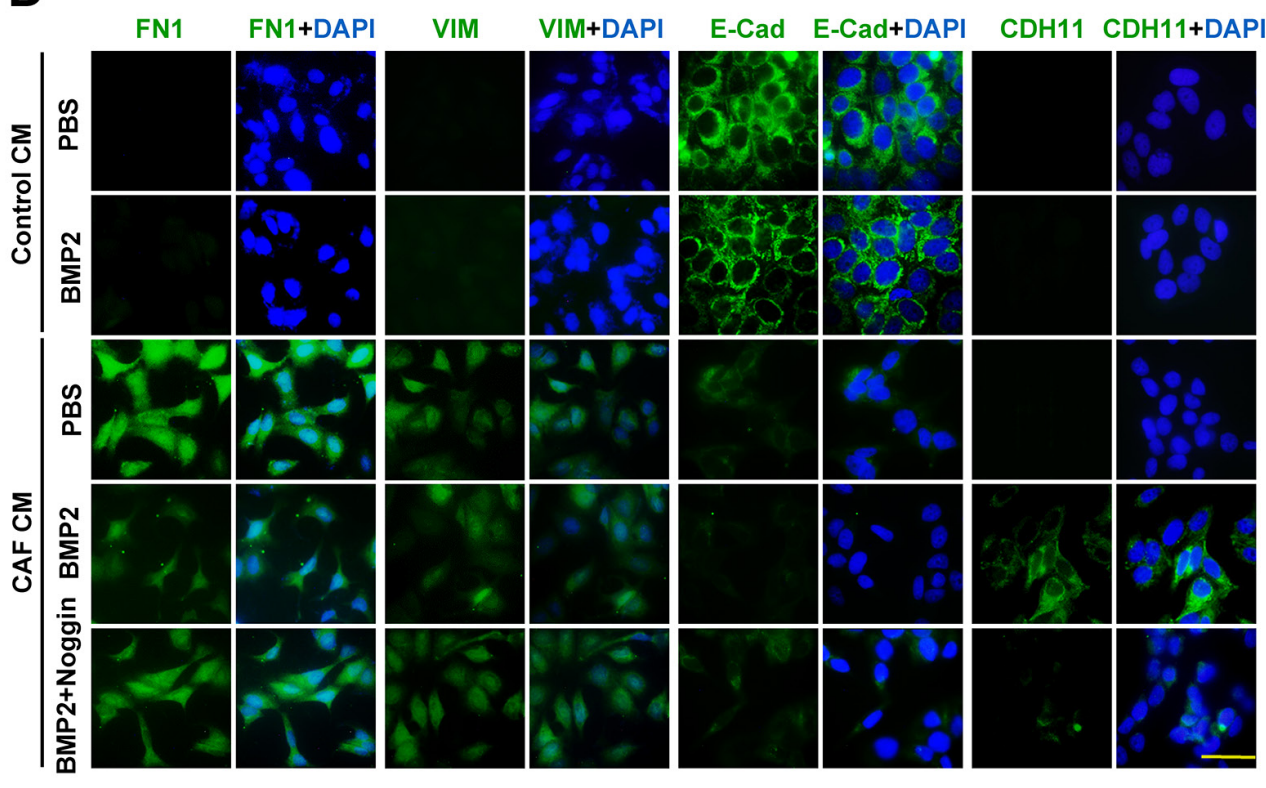

Figure 2: The co-expression of BRGs in breast cancer cells is derived from EMT that has undergone BMP2 induction. MCF-7 and T47D cells were treated as indicated. A. The protein expression of SMAD1, p-SMAD1, SMAD3 and p-SMAD3, as well as EMT markers in the cancer cells was detected by immunoblot. $\beta$-actin was used as a loading control. B. and C. The protein expression of BRGs in cancer cells was detected by immunoblot. $\beta$-actin was used as a loading control. D. The protein expression and localization of FN1, VIM, E-Cad, and CDH11 (green) in MCF-7 cells was detected by immunofluorescence. DAPI staining (blue) indicates the nucleus. Scale bar, $40 \mu \mathrm{m}$. 

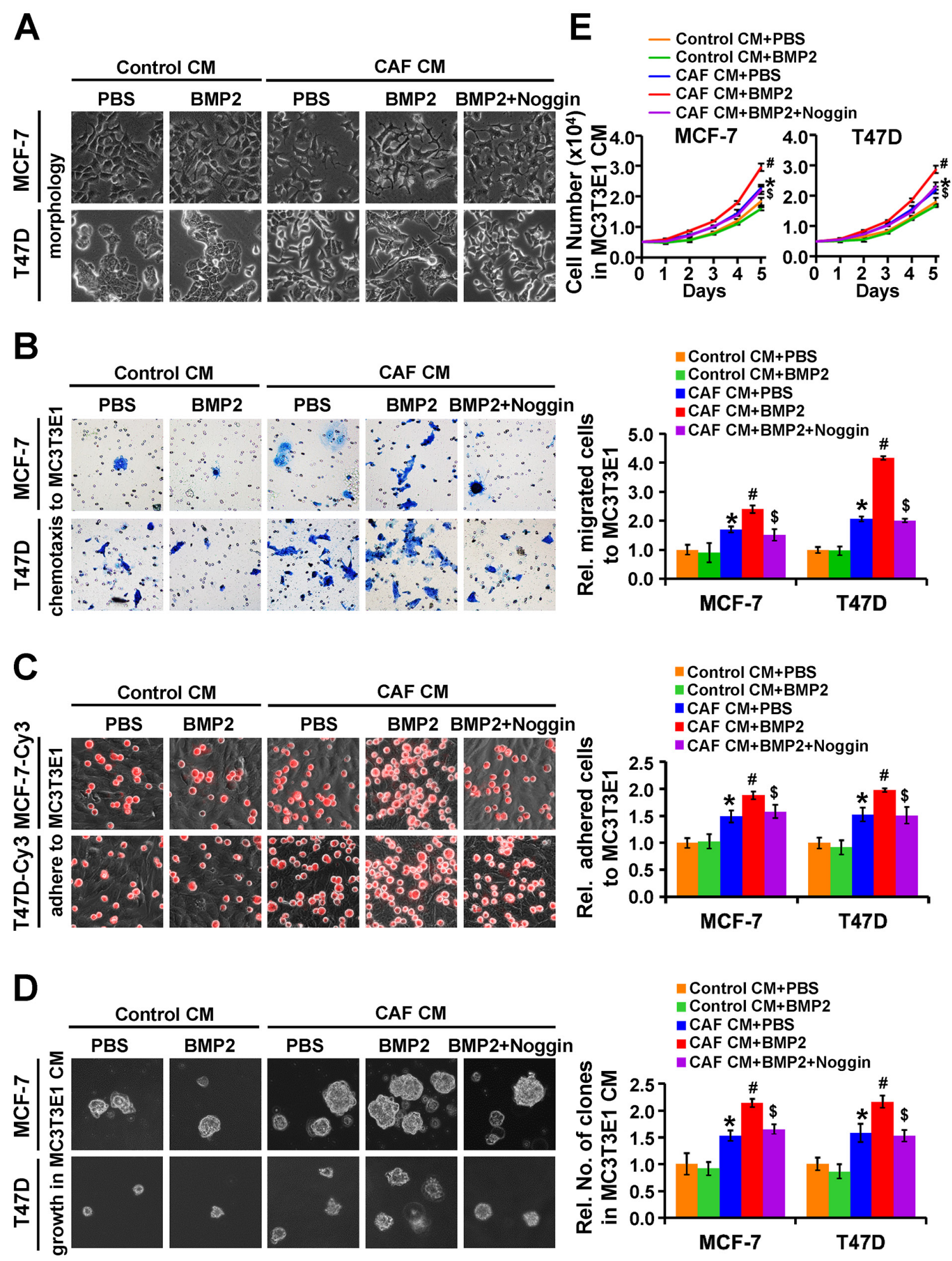

Figure 3: Epithelial cancer cells with co-expression of BRGs induced by CAF/BMP2 signaling gain the advantages of homing to, residing in and growing in an osteoblast-mimic bone microenvironment. MCF-7 and T47D cells were treated as indicated. A. The morphology of the cancer cells. B. The chemotactic migration of cancer cells towards MC3T3E1 cells was assessed by transwell assay. C. The adhesion of cancer cells (red, labeled with Cy3) to MC3T3E1 cells was assessed by putting cancer cells on top of MC3T3E1 cells at 100\% confluence and incubating the co-culture for $30 \mathrm{~min}$. D. The colony formation of cancer cells in soft agar containing MC3T3E1 CM. Magnification: 200X. E. The proliferation of cancer cells in MC3T3E1 CM. Data are presented as the mean \pm S.D. of three independent experiments performed in duplicate. *, $P<0.05$ compared with Control CM plus PBS; $\#, P<0.05$ compared with cells treated with CAF CM plus PBS; $\$, P<0.05$ compared with cells treated with CAF CM plus BMP2. 
A

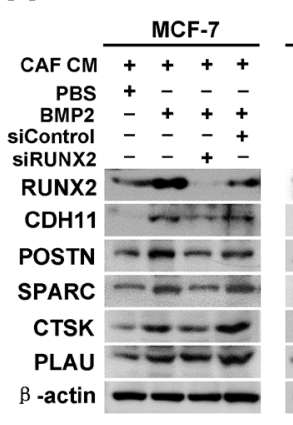

C

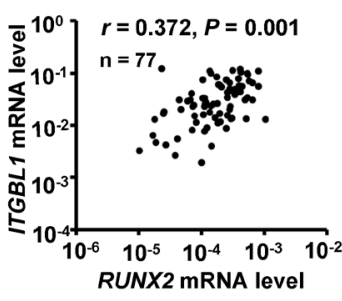

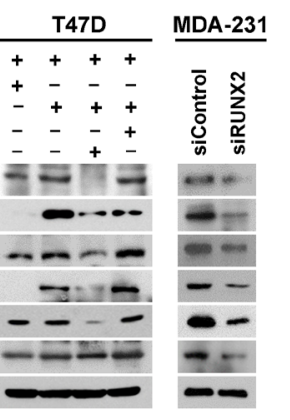

B

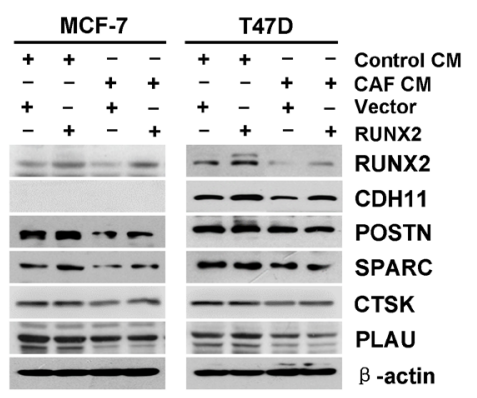

D
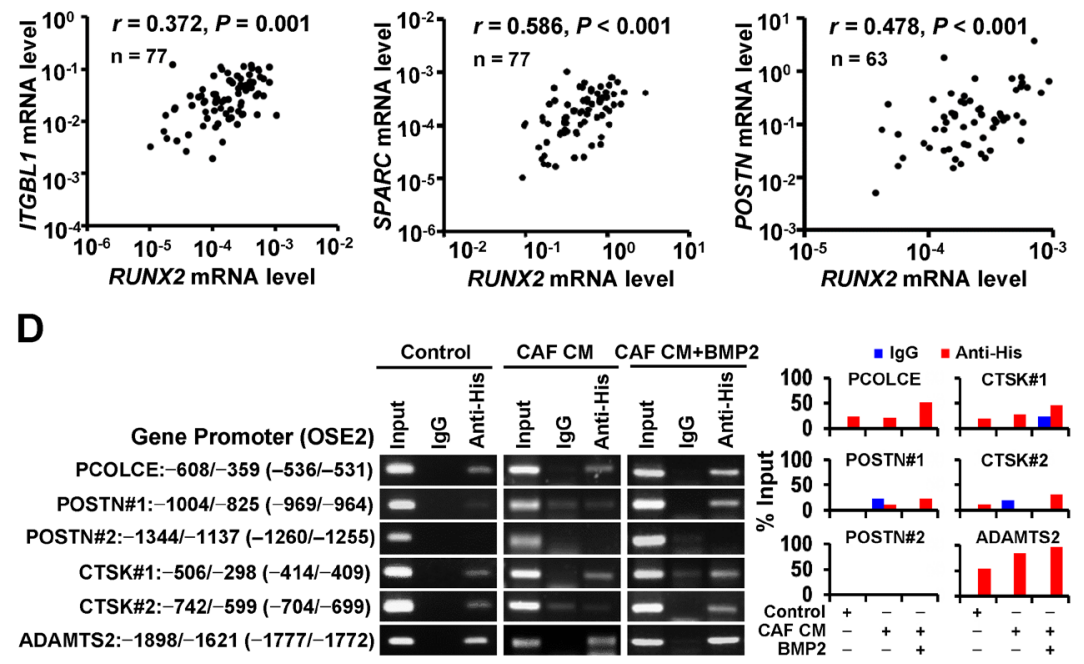

E

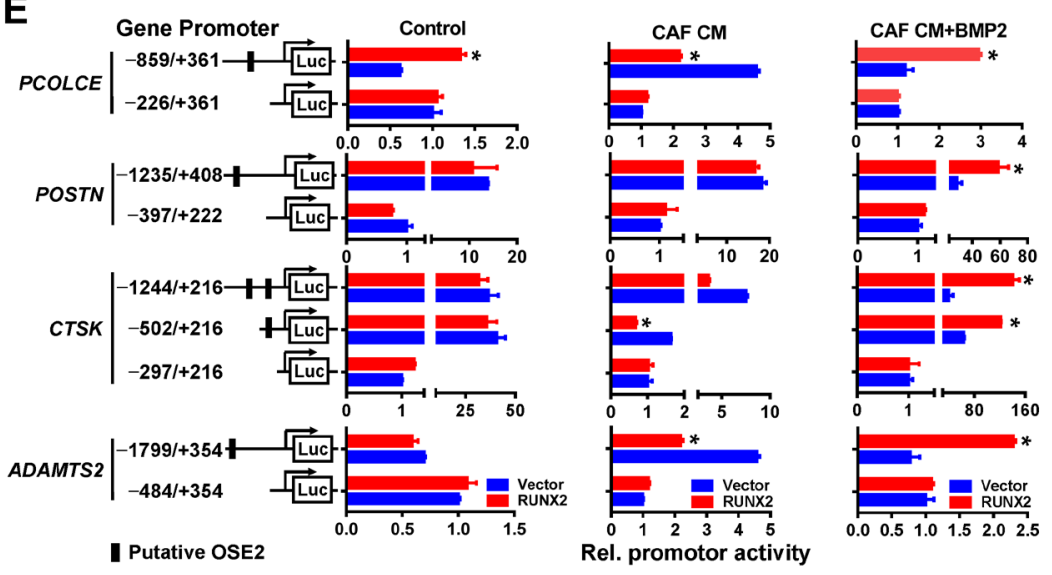

Figure 4: RUNX2 mediates the CAF/BMP2-induced co-expression of BRGs in breast cancer cells through directly and indirectly regulating their transcription. A. The protein levels of BRGs in MCF-7, T47D and MDA-MB-231 cells treated as indicated were detected by immunoblot. $\beta$-actin was used as a loading control. For MCF-7 and T47D cells, RUNX2 was knocked down by RUNX2 siRNA (siRUNX2) transfection in the presence of $100 \mathrm{ng} / \mathrm{mL}$ BMP2 for 3 days after a 3-day culture with CAF CM. For MDAMB-231 cells, RUNX2 was directly knocked down by siRUNX2 transfection. B. The protein levels of BRGs in CAF CM- or Control medium-cultured MCF-7 and T47D cells with the transfection of RUNX2 plasmids or vector control were detected by immunoblot. $\beta$-actin was used as a loading control. C. The correlation of ITGBLI $(\mathrm{n}=77), \operatorname{SPARC}(\mathrm{n}=77)$ or POSTN $(\mathrm{n}=63)$ mRNA levels with RUNX2 mRNA levels in primary breast cancer tissues was analyzed by Pearson's correlation analysis. The mRNA expression of each gene in primary breast cancer tissues was detected by RT-QPCR. D. The binding of RUNX2 to the proximal promoter regions of PCOLCE, POSTN, CTSK, and ADAMTS2 containing or lacking OSE2 in MCF-7 cells transfected with pCDNA3.1-RUNX2-His was assessed by ChIP assay with an anti-His antibody. Isotype IgG was used as a negative control, and the total input was used as a positive control. E. The promoter activity of PCOLCE, POSTN, CTSK and ADAMTS2 containing or lacking OSE2 in MCF-7 cells treated as indicated was detected by dual-luciferase reporter assay. Two independent experiments were performed in duplicate. The data are presented as the mean $\pm \mathrm{SD} . *, P<0.05$ compared with cells transfected with the corresponding vector control. 
Supplementary Figure S4C). Moreover, we observed a positive correlation of the mRNA levels between $R U N X 2$ and BRGs (ITGBL1, SPARC and POSTN) in primary breast cancer tissues (Figure $4 \mathrm{C}$ ). These results suggest that the ectopic co-expression of BRGs in breast cancer cells induced by CAF/BMP2 is mediated through the upregulation of RUNX2, and the activation of the BMP/ SMAD signaling pathway is required for the RUNX2mediated expression of BRGs.

\section{RUNX2 triggers the transcription of a set of BRGs in epithelial breast cancer cells that have undergone CAF/BMP2 induction}

Multiple known transcriptional targets of RUNX2 are included in the set of BRGs, including COL1A1 [25], COL10A1 [26], MMP13 [27] and ITGBL1 [28]. To identify other transcriptional targets of RUNX2 in the set of BRGs, we analyzed the $2 \mathrm{~Kb}$ upstream region of the promoters for the RUNX2 binding sequence "ACCACA" that known as an osteoblast-specific cis-acting element 2 (OSE2). In addition to the known targets of RUNX2, 38 genes contained one or more OSE2 (Table 1). Chromatin immunoprecipitation (ChIP) assays showed the direct bindings of RUNX2 to the OSE2-containing regions of the promoters of PCOLCE (-536/-531), POSTN (-969/-964, -1260/-1255), CTSK (-414/-409, -704/699) and ADAMTS2 (-1777/-1772) in MCF-7 cells with RUNX2-His plasmid transfection, and these bindings were significantly increased (PCOLCE, CTSK, ADAMTS2) or dramatically appeared (POSTN) by CAF/BMP2 induction, but did not affected by CAF CM alone (Figure 4D). Dualluciferase assays showed that RUNX2 overexpression only enhanced PCOLCE promoter activation but not the other promoters in MCF-7 cells, whereas it dramatically activated the promoters of all four genes when the cells were induced with CAF/BMP2 (Figure 4E). Interestingly, the cells treated with $\mathrm{CAF} \mathrm{CM}$ alone showed reduced promoter activity of PCOLCE, CTSK and ADAMTS2 by RUNX2 overexpression (Figure 4E), although the binding of RUNX2 to the promoters of these genes was not significant changed compared with the control cells, indicating that RUNX2 transcriptionally repress its targets of BRGs under the induction of TGF- $\beta / \mathrm{SMAD}$ signaling pathway. The TGF- $\beta / \mathrm{SMAD}$ and BMP/SMAD signaling pathways play opposite roles in regulating the function of RUNX2 transcription factor during these processes: the TGF- $\beta$ /SMAD signaling pathway induces the transcriptionally repressing function of RUNX2, and the BMP/SMAD signaling pathway induces the transcriptionally promoting function of RUNX2. Thus, RUNX2 functions as a master mediator during the transformation of epithelial breast cancer cells into ostomimetic cells under CAF/BMP2 induction, and the expression of BRGs was repressed in the TGF- $\beta / \mathrm{SMAD} /$
RUNX2 signaling pathway, but induced in the BMP/ SMAD/RUNX2 signaling pathway.

\section{RUNX2 is critical for the CAF/BMP2-induced advantages of homing, residing and growing of breast cancer cells in the bone microenvironment}

To further investigate the role of RUNX2 during CAF/BMP2-induced advantages of homing, residing and growing of breast cancer cells in the bone microenvironment, we assessed the chemotaxis, adhesion, anchorage-independent growth and proliferation of MCF7 and T47D cells treated as detailed as above in MC3T3E1 cell- and MG- 63 cell- mimic bone microenvironment. The results revealed that RUNX2 knockdown reversed the osteoblast-like morphology (Figure 5A) and attenuated chemotactic migration (Figure 5B and Supplementary Figure S5A), adhesion (Figure 5C and Supplementary Figure S5B), anchorage-independent growth (Figure 5D and Supplementary Figure S5C) and proliferation (Figure 5E) of MCF-7 and T47D cells induced by CAF/ BMP2. Consistently, RUNX2 knockdown in MDAMB-231 cells directly suppressed chemotaxis, adhesion, anchorage-independent growth and proliferation in MC3T3E1 cell-mimic bone microenvironment (Figures 5A-5E). These results indicate that RUNX2 plays a critical role in the $\mathrm{CAF} / \mathrm{BMP} 2$-induced osteomimetic transformation of epithelial breast cancer cells, which gain the advantages of tending to bone and thriving in the bone microenvironment.

\section{CAF/BMP2/RUNX2-induced osteomimicry enhances the multidrug resistance of breast cancer cells in the tumor and bone microenvironments}

Considering that the ECM, which encoded by BRGs, surrounding breast cancer cells may protect the cells from chemotherapeutic agents, we treated MCF-7 cells as described above in a 3D soft agar culture supplemented with MG-63 CM or Control CM to test the sensitivity to drugs commonly used for breast cancer treatment in clinic, including paclitaxel, cyclophosphamide (CTX), epirubicin and 5-fluorouracil (5-Fu). The results showed that the cells cultured with $\mathrm{CAF} \mathrm{CM}$ showed increased drug resistance in either MG-63 CM or Control CM compared with their corresponding untreated control. Strikingly, the cells cultured with CAF CM plus BMP2 displayed a stronger resistance to cytotoxic drugs compared with those cultured with CAF CM alone in either MG-63 CM or Control CM, especially in MG-63 CM. This effect was weakened by adding noggin or silencing RUNX2 expression (Figures 6A-6B and Supplementary Figures S6A-S6B). These results indicate that RUNX2 plays a critical role in the CAF/BMP2-induced osteomimicry of breast cancer cells, which obtain multidrug resistance in both tumor 
Table 1: BRGs potentially targeted by RUNX2

\begin{tabular}{|c|c|c|c|}
\hline $\begin{array}{l}\text { Gene } \\
\text { symbol }\end{array}$ & $\begin{array}{l}\text { References } \\
\text { sequence number }\end{array}$ & Gene description & $\begin{array}{c}\text { Site of OSE2 relative to } \\
\text { the TSS }\end{array}$ \\
\hline \multirow[t]{2}{*}{$A E B P 1$} & NM_001129 & AE binding protein 1 & $-1395 /-1390$ \\
\hline & & & $-1517 /-1512$ \\
\hline$A D A M 12$ & NM_001288973 & ADAM metallopeptidase domain 12 & $-1962 /-1957$ \\
\hline$A D A M T S 2$ & NM_014244 & $\begin{array}{l}\text { ADAM metallopeptidase with thrombospondin type } 1 \\
\text { motif } 2\end{array}$ & $-1777 /-1772 *$ \\
\hline$B A R X 1$ & NM_021570 & BARX homeobox 1 & $-1561 /-1556$ \\
\hline \multirow[t]{2}{*}{ C1QTNF5 } & NM_015645 & $\mathrm{C} 1 \mathrm{q}$ and tumor necrosis factor related protein 5 & $-1310 /-1305$ \\
\hline & & & $-1912 /-1907$ \\
\hline $\mathrm{CDH} 11$ & NM_001308392 & Cadherin 11 & $-1807 /-1802$ \\
\hline COL1A1 & NM_000088 & Collagen, type I, alpha 1 & $-237 /-232[25]^{* *}$ \\
\hline COL1A2 & NM_000089 & Collagen, type I, alpha 2 & $-604 /-599$ \\
\hline COL5A2 & NM_000393 & Collagen, type V, alpha 2 & $-1472 /-1467$ \\
\hline \multirow[t]{2}{*}{ COL5A3 } & NM_015719 & Collagen, type V, alpha 3 & $-538 /-533$ \\
\hline & & & $-977 /-972$ \\
\hline \multirow[t]{8}{*}{ COL6A2 } & NM_058174 & Collagen type VI, alpha 2 & $-646 /-641$ \\
\hline & & & $-932 /-927$ \\
\hline & & & $-965 /-960$ \\
\hline & & & $-986 /-981$ \\
\hline & & & $-1023 /-1018$ \\
\hline & & & $-1042 /-1037$ \\
\hline & & & $-1085 /-1080$ \\
\hline & & & $-1465 /-1460$ \\
\hline \multirow[t]{3}{*}{ COL6A3 } & NM_004369 & Collagen type VI alpha 3 & $-370 /-365$ \\
\hline & & & $-761 /-756$ \\
\hline & & & $-1299 /-1294$ \\
\hline COL10A1 & NM_000493 & Collagen, type $X$, alpha 1 & $-358 /-353[26]^{* *}$ \\
\hline COMP & NM_000095 & Cartilage oligomeric matrix protein & $-71 /-66$ \\
\hline CTHRC1 & NM_001256099 & Collagen triple helix repeat containing 1 & $-26 /-21$ \\
\hline \multirow[t]{2}{*}{ CTSK } & NM_000396 & Cathepsin K & $-414 /-409 *$ \\
\hline & & & $-704 /-699 *$ \\
\hline \multirow[t]{2}{*}{$D A C T 1$} & NM_001079520 & Dishevelled-binding antagonist of beta-catenin 1 & $-1257 /-1252$ \\
\hline & & & $-1444 /-1439$ \\
\hline \multirow[t]{2}{*}{$\mathrm{DIO} 2$} & NM_013989 & Deiodinase, iodothyronine, type II & $-532 /-527$ \\
\hline & & & $-1750 /-1745$ \\
\hline \multirow[t]{4}{*}{$F B N 1$} & NM_000138 & Fibrillin 1 & $-1186 /-1181$ \\
\hline & & & $-1305 /-1300$ \\
\hline & & & $-1968 /-1963$ \\
\hline & & & (Continued) \\
\hline
\end{tabular}




\begin{tabular}{|c|c|c|c|}
\hline $\begin{array}{l}\text { Gene } \\
\text { symbol }\end{array}$ & $\begin{array}{l}\text { References } \\
\text { sequence number }\end{array}$ & Gene description & $\begin{array}{c}\text { Site of OSE2 relative to } \\
\text { the TSS }\end{array}$ \\
\hline \multirow[t]{2}{*}{ GJB2 } & NM_004004 & Gap junction protein beta 2 & $-366 /-361$ \\
\hline & & & $-1732 /-1727$ \\
\hline ITGBL1 & NM_004791 & Integrin beta-like 1 & $-793 /-788[28]^{* *}$ \\
\hline \multirow[t]{2}{*}{$L O X$} & NM_002317 & Lysyl oxidase & $-1033 /-1028$ \\
\hline & & & $-1048 /-1043$ \\
\hline \multirow[t]{2}{*}{$L O X L 2$} & NM_002318 & Lysyl oxidase-like 2 & $-1135 /-1130$ \\
\hline & & & $-1646 /-1641$ \\
\hline$M M P 3$ & NM_002422 & Matrix metallopeptidase 3 & $-978 /-973$ \\
\hline \multirow[t]{2}{*}{$M M P 13$} & NM_002427 & Matrix metallopeptidase 13 & $-143 /-138[27]^{* *}$ \\
\hline & & & $-136 /-131[27]^{* *}$ \\
\hline \multirow[t]{2}{*}{ NOX4 } & NM_001143837 & NADPH Oxidase 4 & $-280 /-275$ \\
\hline & & & $-1526 /-1521$ \\
\hline$O G N$ & NM_014057 & Osteoglycin & $-1821 /-1816$ \\
\hline PCOLCE & NM_002593 & Procollagen c-endopeptidase enhancer & $-536 /-531 *$ \\
\hline$P D P N$ & NM_006474 & Podoplanin & $-170 /-165$ \\
\hline$P L A U$ & NM_002658 & Plasminogen activator & $-1724 /-1719$ \\
\hline$P L X D C 2$ & NM_032812 & Plexin domain containing 2 & $-723 /-718$ \\
\hline \multirow[t]{2}{*}{ POSTN } & NM_006475 & Periostin & $-969 /-964 *$ \\
\hline & & & $-1260 /-1255^{\#}$ \\
\hline$S F R P 2$ & NM_003013 & Secreted frizzled-related protein 2 & $-1749 /-1744$ \\
\hline \multirow[t]{4}{*}{ SPARC } & NM_001309443 & Secreted protein acidic and cysteine rich & $-866 /-861$ \\
\hline & & & $-1203 /-1198$ \\
\hline & & & $-1795 /-1790$ \\
\hline & & & $-1874 /-1869$ \\
\hline SPOCK & NM_004598 & $\begin{array}{l}\text { Sparc/osteonectin, cwcv and kazal-like domains } \\
\text { proteoglycan }\end{array}$ & $-346 /-341$ \\
\hline$T G F B I$ & NM_000358 & Transforming growth factor beta-induced & $-1296 /-1291$ \\
\hline THY1 & NM_001311162 & Thy-1 cell surface antigen & $-1751 /-1746$ \\
\hline TIMP2 & NM_003255 & TIMP metallopeptidase inhibitor 2 & $-588 /-583$ \\
\hline
\end{tabular}

Note: *, identified binding site of RUNX2 in this study; **, identified target of RUNX2 in the reference's report; \#, identified non-binding site of RUNX2 in this study.

and bone microenvironments, and that RUNX2 depletion efficiently abolishes these effects.

\section{DISCUSSION}

Organ-specific metastasis depends on the intrinsic molecular characteristics of cancer cells, referred to as "pre-selected" seeds [29], and a suitable microenvironment in target organs, which is created by signals released from metastatic cancer cells, referred to as a "pre-metastatic niches" [30]. Accumulating evidence suggests that cancer cells with osteomimetic features that ectopically co-expressing BRGs have an increased bone metastatic potential [2]. In the present study, we identified a set of BRGs by analyzing co-expressed genes in primary breast cancer tissues based on our gene expression profiling dataset. The set of BRGs primarily includes genes that encode functional bone remodeling-related 
A

MCF-7+CAF CM+BMP2

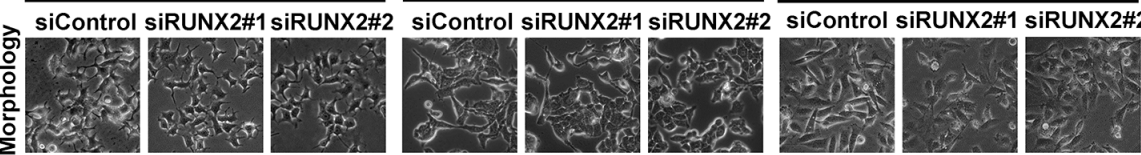

B

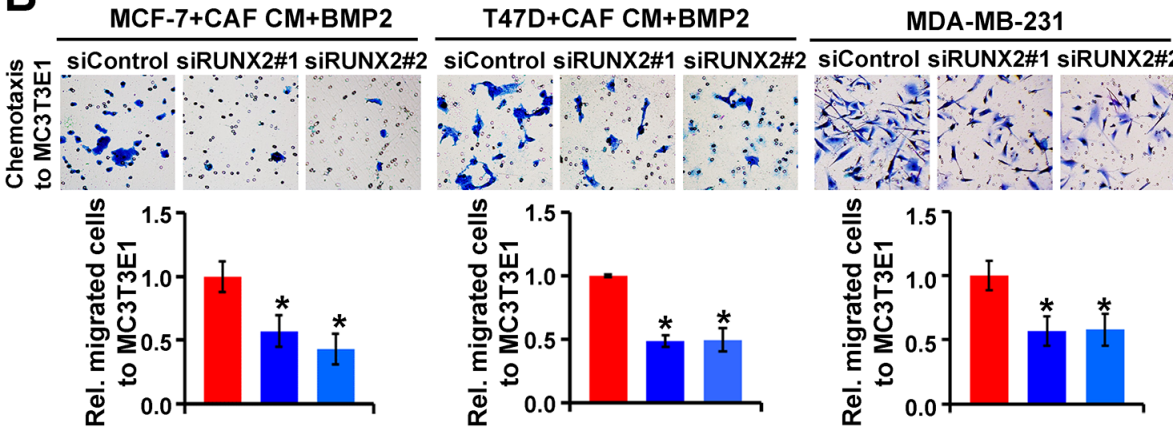

C

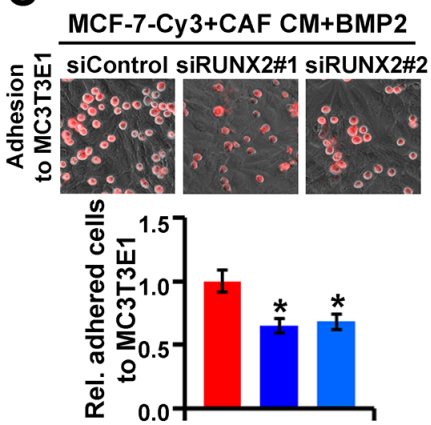

T47D-Cy3+CAF CM+BMP2

MDA-MB-231-Cy3
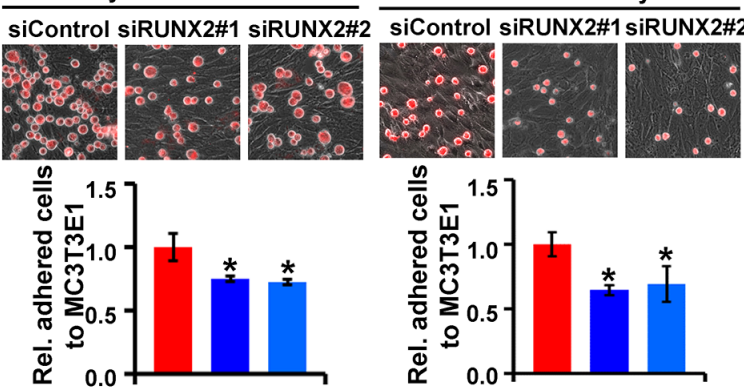

D
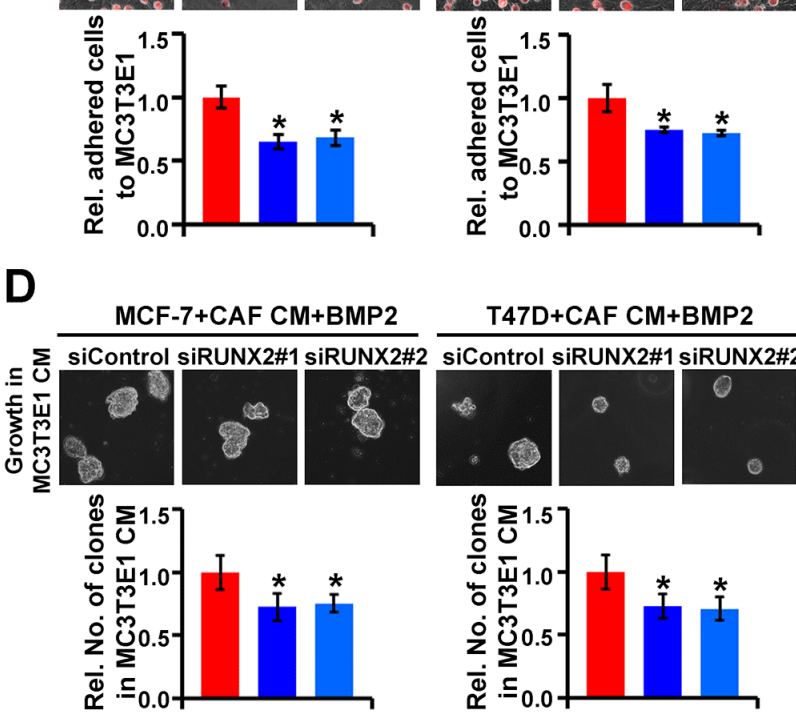

$\mathbf{E}$

MCF-7+CAF CM+BMP2

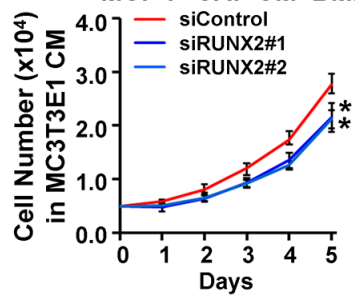

T47D+CAF CM+BMP2
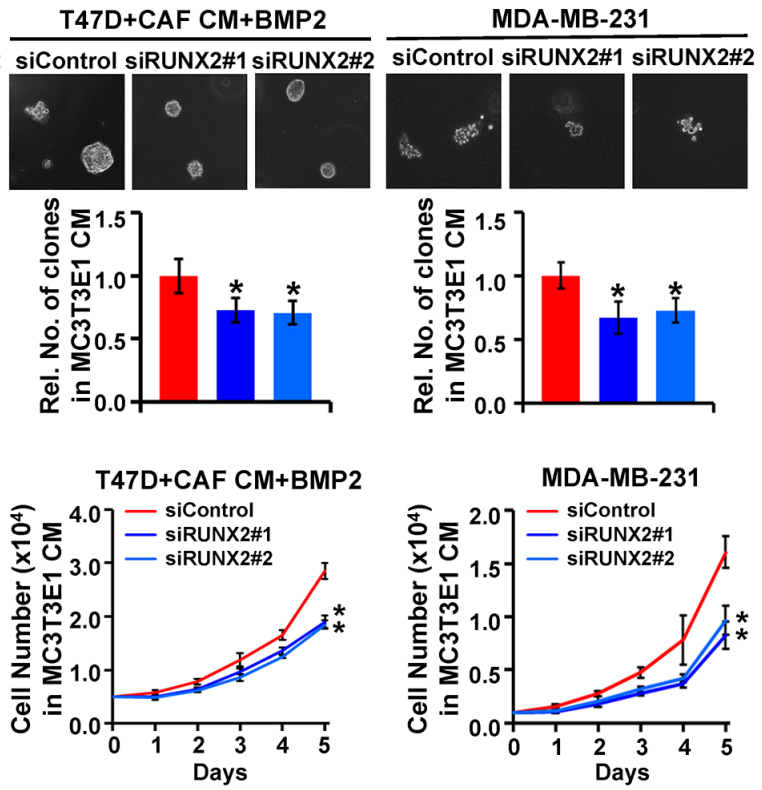

Figure 5: RUNX2 is critical for the CAF CM/BMP2-induced advantages of homing, residing and growing of breast cancer cells in an osteblast-mime bone microenvironment. MCF-7, T47D and MDA-MB-231 cells were treated as indicated. A. The morphology of cancer cells. B. The chemotactic migration of cancer cells towards MC3T3E1 cells was assessed by transwell assay. C. The adhesion of cancer cells (red, labeled with Cy3) to MC3T3E1 cells was assessed by putting cancer cells on top of MC3T3E1 cells at $100 \%$ confluence and incubating the co-culture for $30 \mathrm{~min}$. D. The colony formation of cancer cells in soft agar with MC3T3E1 CM. Magnification: 200X. E. The proliferation of cancer cells in MC3T3E1 CM. Three independent experiments were performed in duplicate. The data are presented as the mean $\pm \mathrm{SD}$. *, $P<0.05$ compared with the corresponding siControl. 
proteins, some of which are known to be involved in bone metastasis [2] and are included in the "bone metastasis gene signature" or "osteoblast-like gene expression signature" [31]. Importantly, we found that the set of BRGs was more highly co-expressed in breast cancer tissues compared with normal breast tissues. This result suggests that the ectopic co-expression of these BRGs is commonly existed in breast cancer tissues, which may explain why breast cancers preferentially metastasize to bone, with an incidence of bone metastasis as high as $80 \%$ in advanced breast cancer patients [32]. Moreover, we also found that these BRGs were more highly co-expressed in bone metastatic tissues than in metastatic tissues of other organs. This result supports the hypothesis that the osteomimetic feature confers on cancer cells the properties to selectively metastasize to bone.

Although it is known that the osteomimetic feature with ectopic expression of BRGs enables cancer cells to metastasize to bone, the pathogenesis of breast cancer osteomimicry remains unknown. Physiologically, the expression of BRGs initially appears during development, metabolism and the repair of damage of bone, and is derived from the directed differentiation of bone marrow mesenchymal stem cells under the induction of growth factors. BMP2 is an important growth factor for inducing the differentiation of bone marrow mesenchymal cell into osteoblasts through regulating the osteoblast-specific transcription factor RUNX2 to trigger the expression of BRGs [33]. BMP2 has been reported to be highly expressed in breast cancer tissues and is related to breast cancer bone metastasis $[19,20]$. CAFs, the most abundant stromal cells in breast cancer tissues, can induce breast cancer cell EMT through paracrine TGF- $\beta$ signaling [12]. Cancer cells that have undergone EMT exhibit multilineage differentiation potential similar to mesenchymal stem cells [16]. Therefore, we speculated that the ectopic co-expression of BRGs in breast cancers may be derived from epithelial cancer cells that have undergone EMT followed by BMP2 induction in the CAF-enriched tumor microenvironment. Indeed, by performing in vitro experiments that mimic the tumor microenvironment, we demonstrated that the CAF/BMP2/RUNX2 signaling axis transducted from stromal cells to cancer cells can confer breast cancer cells to obtain an osteomimetic feature that function as pre-selected bone metastatic seeds. Our results also provide insights regarding the origin of cancer tissue heterogeneity, which may arise due to stromal cell-induced EMT and stemness of cancer cells, followed by multilineage differentiation depending on the specific growth factor signals in the tumor microenvironment.

A

Control CM
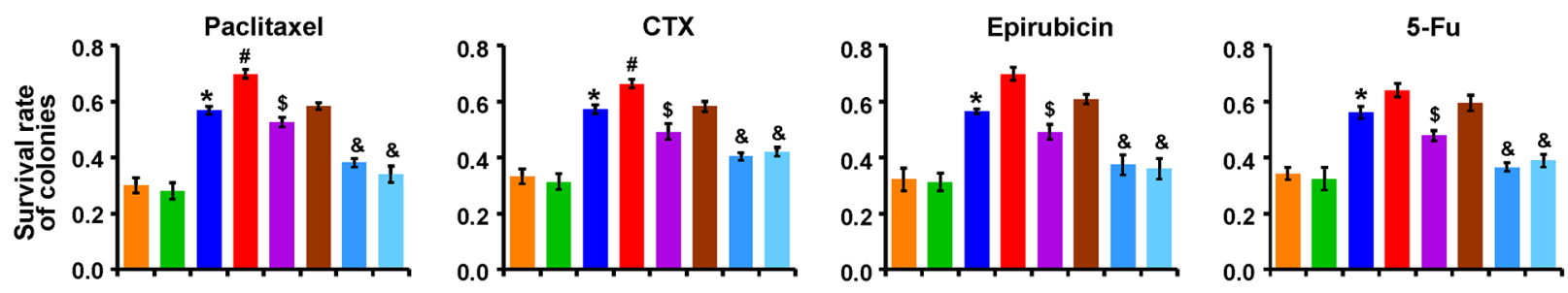

B
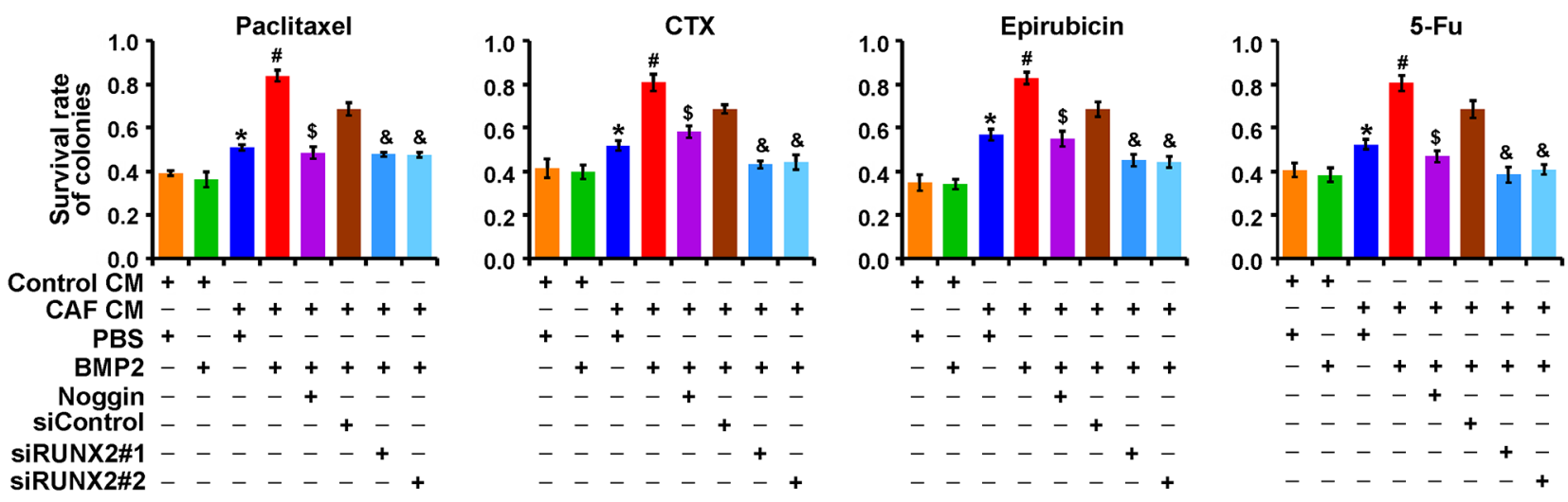

Figure 6: CAF/BMP2/RUNX2-induced co-expression of BRGs in breast cancer cells enhances multidrug resistance. Quantification of live colonies of MCF-7 cells treated as indicated in soft agar with Control CM A. or MG-63 CM B. after drug treatment relative to untreated control cells. Three independent experiments were performed in duplicate. The data are presented as the mean $\pm \mathrm{SD}$. *, $P<0.05$ compared with cells treated with Control CM plus PBS; \#, $P<0.05$ compared with cells treated with CAF CM plus PBS; $\$, P<0.05$ compared with cells treated with CAF CM plus BMP2; \&, $P<0.05$ compared with cells treated with CAF CM/BMP2 plus siControl. 
The formation of bone metastatic lesions is a complex process in which metastatic cancer cells interact with the bone matrix, osteoblasts, and osteoclasts to result in osteolytic or osteoblastic lesions by interrupting the balance of osteoblastogenesis and osteoclastogenesis [34]. Most of the ectopically expressed BRGs in breast cancer cells contribute to bone remodeling and bone metastasis. RUNX2, the master mediator of bone remodeling, is responsible for not only the activation of osteoblast differentiation [35] but also the regulation of osteoclast differentiation [36]. RUNX2 also contributes to the inhibition of osteoblast differentiation in breast cancer bone metastasis [37], depending on the primary tumor microenvironment and the bone microenvironment induced by metastatic cancer cells. CDH11, also known as OB-cadherin, is constitutively expressed in marrow stromal/osteoblastic cells and mediates homophilic cell adhesion [38]. CDH11 has been reported to be overexpressed in aggressive breast cancers [39] and bone metastatic breast cancer cells [8]. CDH11 selectively promotes bone metastatic homing and colonizatione of breast cancer cells and induces osteoclastogenesis through mediating interactions between breast cancer cells and marrow stromal/osteoblastic cells [40]. In both primary and metastatic cancer tissues, the ECM surrounding cancer cells not only supports cell survival, proliferation and progression, but also protects cells from chemotherapeutic agents, therefore conferring resistance to multidrug therapy $[41,42]$. Our data support the hypothesis (Figure 7) that breast cancer cells with overexpression of BRGs induced by $\mathrm{CAF} / \mathrm{BMP} 2$ signaling acquire the abilities of homing to, residing in and growing in the bone microenvironment, as well as resistance to multidrug therapies commonly used for breast cancer treatment in the clinic.

RUNX2 is post-translationally activated during the early stage of osteoblast differentiation to transcriptionally regulate multiple genes associated with mineralization in mature osteoblasts [43]. The activity, stability, and interactions with transcriptional co-regulators and chromatin remodeling proteins of RUNX2 under osteogenic signal stimulation are dependent on posttranslational modifications, including phosphorylation, ubiquitination, and acetylation [44]. The post-translational modification of RUNX2 might be used to explain the inconsistencies that its mRNA and protein levels exhibit the same trend changes upon CAF/BMP2 induction but opposite changes upon induction with CAF alone (data not shown). RUNX2 lies downstream of the TGF- $\beta /$ SMAD and $\mathrm{BMP} / \mathrm{SMAD}$ signaling pathways to transcriptionally regulate the expression of various BRGs and ECM remodeling-related genes [45]. In addition to the known targets of RUNX2, including COL1A1 [25], COL10A1 [26] and ITGBL1 [28], we identified PCOLCE, POSTN, $C T S K$ and $A D A M T S 2$ as novel transcriptional targets of RUNX2 from multiple candidates in the set of BRGs. Among them, PCOLCE [46], POSTN [47] and CTSK [48] have been functionally validated as contributors to bone metastasis. Importantly, we found that RUNX2 differentially regulates its target genes in response to different microenvironmental stimuli: RUNX2 transcriptionally activates these genes in epithelial cancer cells that have undergone the CAF/BMP2-induced osteomimicry transformation but transcriptionally suppresses these targets in epithelial cancer cells that have undergone CAF-induced EMT, in which TGF- $\beta /$ SMAD signaling induces transcriptional suppression function of RUNX2. Thus, RUNX2 functions as a master mediator during the transformation of epithelial breast cancer cells into osteomimetic cells under the induction of $\mathrm{CAF} /$ BMP2, and the expression of BRGs is repressed by the TGF- $\beta /$ SMAD/RUNX2 signaling pathway but induced by the BMP/SMAD/RUNX2 signaling pathway. These results are consistent with the regulatory machinery of RUNX2 for skeletal gene expression [49] and the antagonism of BMP 2 and TGF- $\beta$ signaling during stem cell maintenance and osteogenesis [50]. Our study provides insight into the pleiotropic regulatory role of transcription factors, e.g., RUNX2 in cell fate determination and the regulation of differentiation during tissue development and tumor progression.

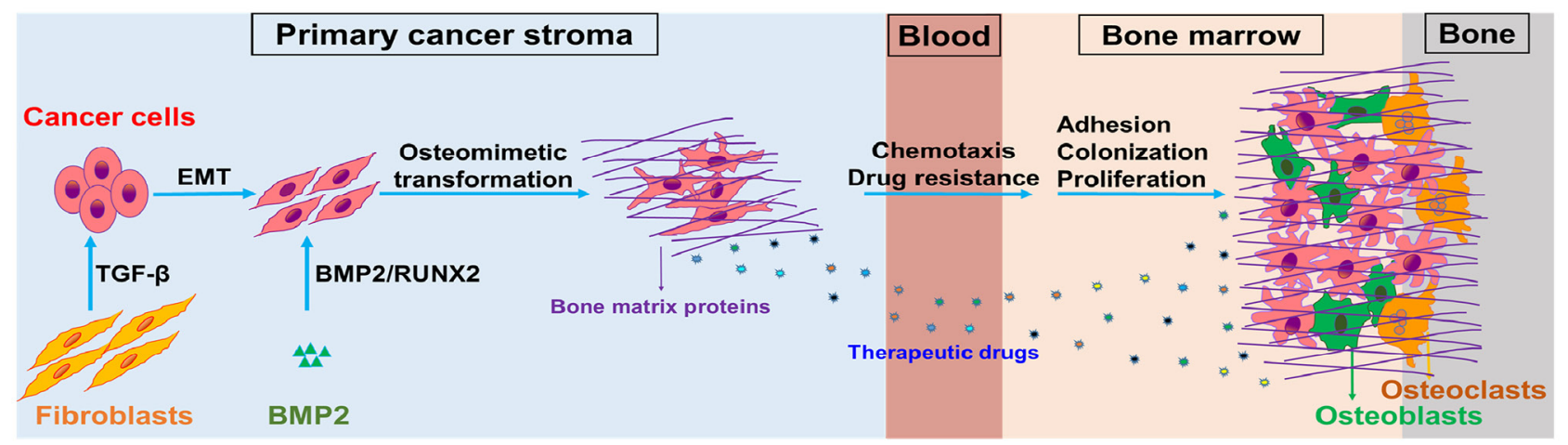

Figure 7: A schematic of the osteomimicry transdifferentiation of breast cancer cells. CAF/BMP2 signaling in the primary cancer stroma confers an osteomimetic feature with ectopic co-expression of BRGs upon cancer cells, which acquires the advantages of chemotaxis, adhesion, colonization, proliferation and multidrug resistance in the bone microenvironment. 
Indeed, this study lacks an appropriate animal model to validate our clinical and in vitro data due to experimental limitations. EMT process might be a transient and reversible phenomenon that is difficult to maintain especially in the complex internal environment in vivo [51]. In addition, we focused on EMT, not stem cells, based on the link between EMT and CSCs [14], whether the expression pattern of BRGs induced by EMT/BMP2 is similar in BMP2-stimulated CSCs will be investigated in future studies. Furthermore, besides the known target genes and those validated in this study (PCOLCE, POSTN, CTSK and ADAMTS2), multiple candidate targets of RUNX2 (Table 1) were not functionally validated in terms of their regulation by RUNX2 and contribution to metastatic bone disease. The functional elucidations of RUNX2 transcriptional targets would significantly improve the understanding of the propensity of certain cancers to metastasize to the skeleton.

In summary, our data demonstrated that the osteomimetic feature of breast cancer with ectopic coexpression of BRGs is obtained from the epithelial cancer cells that undergo stromal cell-induced EMT followed by BMP2-induced transcriptional activation function of RUNX2 in the tumor microenvironment. Our findings provide functional and mechanistic evidence to explain how advanced breast cancers commonly metastasize to bone and suggest a potential strategy for the prevention and treatment of breast cancer bone metastatic disease by targeting the EMT/BMP2/RUNX2 pathway and disrupting the interaction between cancer cells and their microenvironment.

\section{MATERIALS AND METHODS}

\section{Gene expression profiling datasets}

The gene expression profiling datasets of 9 normal breast cancer tissues and 49 primary breast cancer tissues were generated in our previous study [21]. The gene expression profiling datasets of 29 human breast cancer metastatic tissues, including 4 lung metastases, 15 brain metastases and 10 bone metastases, were generated by Zhang XH, et al. (GEO accession number GSE14017) [20]. GeneCards (http://www.genecards. org/) and NCBI-PubMed (http://www.ncbi.nlm.nih.gov/ pubmed/) databases were used to select BRGs that encode osteoblast-specific transcription factors, osteoblast-specific adhesion molecules, bone matrix proteins, bone matrixdegrading enzymes, and growth factors that regulate bone remodeling and bone-related disease.

\section{Tissue specimens}

Total 77 primary breast cancer tissues were collected from patients with invasive ductal carcinoma who had undergone a mastectomy at the Tianjin Medical University
Cancer Institute \& Hospital (TMUCIH; Tianjin, China). All tissue samples were snap-frozen in liquid nitrogen and stored at $-80^{\circ} \mathrm{C}$. The use of specimens in this study was approved by the Institutional Review Board of TMUCIH, and written consent was obtained from all participants.

\section{Cell culture and induction}

The human breast cancer cell lines T47D and MDA-MD-231 were cultured in RPMI 1640 (Invitrogen, Carlsbad, CA, USA), and the human breast cancer cell line MCF-7 and the osteosarcoma cell line MG-63 were cultured in Dulbecco's modified Eagle's medium nutrient mixture basal medium (Invitrogen). The mouse preosteoblastic cell line MC3T3E1 was cultured in minimum essential medium alpha (Invitrogen). The osteoblast differentiation of MC3T3E1 cells was induced with $10 \mathrm{mM} \beta$-glycerol phosphate (Sigma, St. Louis, MO, USA) and $50 \mu \mathrm{g} / \mathrm{mL}$ L-ascorbic acid 2-phosphate (Sigma) for 14 days. Then, cancer cells were co-cultured with differentiated MC3T3E1 cells and MG-63 cells or cultured in their CM diluted with three volumes of fresh normal medium. The isolation of CAFs from primary breast cancer tissues, the collection of CM from low passage CAFs, and the EMT induction of breast cancer cells with CAF CM were performed as previously described [12]. To induce an osteomimetic phenotype of MCF-7 and T47D cells that had undergone EMT in response to CAF CM, the cells were treated with $100 \mathrm{ng} / \mathrm{mL}$ recombinant human BMP2 (Sigma) in the cultured medium for $72 \mathrm{~h}$. A total of $500 \mathrm{ng} / \mathrm{mL}$ recombinant human noggin (BMP antagonist; R\&D Systems, Minneapolis, MN, USA) was used to reverse the effects induced by BMP2. Phosphate-buffered saline (PBS) was added at an equal volume in a parallel test as a control. All cell cultures were supplemented with 10\% fetal bovine serum (FBS; Invitrogen), 100 $\mathrm{U} / \mathrm{mL}$ penicillin and $100 \mu \mathrm{g} / \mathrm{mL}$ streptomycin at $37^{\circ} \mathrm{C}$ in a humidified $5 \% \mathrm{CO}_{2}$ incubator.

\section{RT-QPCR}

RNA extraction from tissues or cells, reverse transcription (RT), quantitative polymerase chain reaction (QPCR) and the quantification of target gene expression were performed as previously described [52]. The sequences of primers and TaqMan probes for RT-QPCR are listed in Supplementary Table S1.

\section{Immunoblot and immunofluorescence}

Immunoblot and immunofluorescence were performed as described previously [12]. The primary and secondary antibodies used in immunoblot and/or immunofluorescence are described in Supplementary Table S2. Band intensities of immunoblot were quantified using Image-Pro Plus 6.0. 


\section{Plasmids and small interfering RNA transfection}

To silence $R U N X 2$ expression in CAF/BMP2-induced MCF-7 and T47D cells or MDA-MB-231 cells, three small interfering RNAs (siRNAs) targeting unique RUNX2 mRNA sequences (siRUNX2) were synthesized (RiboBio Co., Guangzhou, China). Two of the three siRUNX2 with optimal knockdown efficiency as determined by RT-QPCR and immunoblot were selected for the transient RUNX2 knockdown experiments. Non-targeting siRNA (siControl) was used as a control. To force RUNX2 expression in MCF-7 and T47D cells, pcDNA3.1-RUNX2-His plasmid was transiently transfected into the cells, and the empty vector was used as a control. Plasmid and siRNA transfections were performed using Lipofectamine 2000 (Invitrogen) according to the manufacturer's instructions.

\section{Chemotactic migration assay}

Chemotactic migration of breast cancer cells towards an osteoblast-mimic bone microenvironment in vitro was assessed using transwell inserts $(8-\mu \mathrm{m}$ pore size; BD Biosciences, CA, USA) in a 24-well plate. MC3T3E1 or MG-63 cells were pre-seeded in the lower chamber with culture medium containing $10 \%$ FBS for $24 \mathrm{~h}$ to mimic the bone microenvironment. Breast cancer cells $\left(2.5 \times 10^{4}\right.$ MDA-MB-231 cells or $1 \times 10^{5}$ MCF- 7 or T47D cells) in $500 \mu \mathrm{L}$ of serum-free medium were seeded in the upper chamber. The cells were allowed to migrate for an appropriate time. The cancer cells that migrated toward the osteoblasts were counted as described previously [12].

\section{Heterogeneous cell-cell adhesion assay}

The heterogeneous adhesion of breast cancer cells to osteoblasts in vitro was assessed by seeding Cy3-labeled MCF-7, T47D, or MDA-MB-231 cells on pre-inoculated MC3T3E1 or MG-63 cells at nearly $100 \%$ confluence in a 24-well plate for the appropriate time. The non-adherent cells were removed, and washed with PBS. Then, the adherent cells were fixed with $4 \%$ paraformaldehyde and imaged using a fluorescence microscope. The nonadherent cancer cells were counted, and the percentage of adherent cells was calculated as (1- non-adherent cells / total inoculated cells) $\times 100 \%$.

\section{Cell proliferation assay}

The proliferation ability of breast cancer cells in the osteoblast-mimic bone microenvironment in vitro was assessed by cell growth curve analysis as previously described [28].

\section{Soft agar colony formation assay}

Anchorage-independent cell growth was assessed using a soft agar colony formation assay. A $0.6 \%$ agarose gel in serum-free normal medium was used as the bottom gel in 6-well plates. A total of $5 \times 10^{3}$ cells were suspended in $0.3 \%$ agarose in MC3T3E1 CM or MG-63 CM with $10 \%$ FBS and placed on top of the bottom gel. The cells were cultured for 14 days and were fed twice a week with $0.5 \mathrm{~mL}$ of fresh CM. Cell colonies were imaged using a phase contrast microscope, and colonies greater than 50 $\mu \mathrm{m}$ in diameter were counted.

\section{Drug sensitivity testing}

In the culture system of soft agar colony formation assay at day nine, the cells were treated with $1 \mu \mathrm{g} / \mathrm{mL}$ paclitaxel, $10 \mu \mathrm{g} / \mathrm{mL}$ epirubicin, $10 \mu \mathrm{g} /$ $\mathrm{mL} \mathrm{CTX} \mathrm{or} 50 \mu \mathrm{g} / \mathrm{mL} 5$-Fu for 5 days. Cell colonies were stained with 3-(4,5-dimethylthiazol-2-yl)-2,5diphenyltetrazolium bromide (MTT; $5 \mathrm{mg} / \mathrm{mL}$ in PBS), then imaged and counted using a microscope. Cellular drug sensitivity was determined by comparing the colony number of drug-treated cells to that of nontreated control cells.

\section{ChIP assay}

Cancer cells transfected with pcDNA3.1-RUNX2His were lysed and sonicated until chromatin DNA was fragmented to 300 to $500 \mathrm{bp}$. RUNX2-bound DNA was immunoprecipitated with an anti-His antibody (Cell Signaling Technology, Danvers, MA, USA), and the target fragments containing OSE2 were identified by PCR amplification using the primers listed in Supplementary Table S3. IgG was used as a negative control in the immunoprecipitations, and input DNA precipitated without antibody was used as a positive control. The ChIP-PCR products were detected by agarose gel electrophoresis and quantified by integrating optical density using Image-Pro Plus 6.0.

\section{Dual-luciferase reporter assay}

To obtain luciferase reporter constructs for candidate RUNX2 target gene promoters containing or lacking the OSE2, the promoter sequences were amplified from human genomic DNA using the primers listed in Supplementary Table S3 and subsequently inserted into the pGL3-basic vector (Promega, Madison, WI, USA) between the KpnI and XhoI restriction sites. To assess promoter activation, the cells were seeded in 24-well plates and grown to $80 \%$ confluence. RUNX2-expressing plasmids or vector controls were co-transfected with the promoter constructs and the internal control pRL-TK into the cells. Firefly and Renilla luciferase activities were measured $48 \mathrm{~h}$ after transfection using a Dual-Luciferase Reporter Assay System (Promega). The relative promoter activation is presented as the ratio of firefly to Renilla luciferase activity. 


\section{Statistical analyses}

Pearson's correlation analysis was used to assess the correlation of mRNA expression between genes based on the gene expression profiling datasets and RT-QPCR data of breast cancer tissues. Chi-square test, Fisher's exact test or Wilcoxon rank sum test was applied to evaluate the differences of BRG mRNA expression between normal tissues and primary breast cancer tissues or between bone metastatic tissues and metastatic tissues in other organ. All in vitro experimental data are presented as the mean \pm standard deviation (SD). Student's $t$-test was used to compare the differences between the experimental group and the control group. $P<0.05$ was considered significant.

\section{ACKNOWLEDGMENTS}

This work was supported by the National Natural Science Foundation of China (No. 30872518, No. 81272357, No. 81472680 and No. 81672894), the Major Program of Applied Basic Research Projects of Tianjin (No. 09JCZDJC19800 and No. 13JCZDJC30100), the National Science and Technology Support Projects (No. 2015BAI12B15) and the Major Programs of Anti-cancer Projects of Tianjin (No. 12ZCD2SY16000).

\section{CONFLICTS OF INTEREST}

The authors declare no conflict of interest.

\section{REFERENCES}

1. Kozlow W, Guise TA. Breast cancer metastasis to bone: mechanisms of osteolysis and implications for therapy. J Mammary Gland Biol Neoplasia. 2005; 10: 169-80.

2. Bellahcene A, Bachelier R, Detry C, Lidereau R, Clezardin $\mathrm{P}$, Castronovo V. Transcriptome analysis reveals an osteoblast-like phenotype for human osteotropic breast cancer cells. Breast Cancer Res Treat. 2007; 101: 135-48.

3. Kang Y. Dissecting Tumor-Stromal Interactions in Breast Cancer Bone Metastasis. Endocrinol Metab (Seoul). 2016; 31: 206-12.

4. Kovacheva M, Zepp M, Berger SM, Berger MR. Sustained conditional knockdown reveals intracellular bone sialoprotein as essential for breast cancer skeletal metastasis. Oncotarget. 2014; 5: 5510-22.

5. Reufsteck C, Lifshitz-Shovali R, Zepp M, Bauerle T, Kubler D, Golomb G, Berger MR. Silencing of skeletal metastasisassociated genes impairs migration of breast cancer cells and reduces osteolytic bone lesions. Clin Exp Metastasis. 2012; 29: 441-56.

6. Kapoor P, Suva LJ, Welch DR, Donahue HJ. Osteoprotegrin and the bone homing and colonization potential of breast cancer cells. J Cell Biochem. 2008; 103: 30-41.
7. Ribeiro N, Sousa SR, Brekken RA, Monteiro FJ. Role of SPARC in bone remodeling and cancer-related bone metastasis. J Cell Biochem. 2014; 115: 17-26.

8. Tamura D, Hiraga T, Myoui A, Yoshikawa H, Yoneda T. Cadherin-11-mediated interactions with bone marrow stromal/osteoblastic cells support selective colonization of breast cancer cells in bone. Int J Oncol. 2008; 33: 17-24.

9. Rucci N, Teti A. Osteomimicry: how tumor cells try to deceive the bone. Front Biosci (Schol Ed). 2010; 2: 907-15.

10. Li XQ, Lu JT, Tan CC, Wang QS, Feng YM. RUNX2 promotes breast cancer bone metastasis by increasing integrin alpha5-mediated colonization. Cancer Lett. 2016; 380: 78-86.

11. Gordon JA, Sodek J, Hunter GK, Goldberg HA. Bone sialoprotein stimulates focal adhesion-related signaling pathways: role in migration and survival of breast and prostate cancer cells. J Cell Biochem. 2009; 107: 1118-28.

12. Yu Y, Xiao CH, Tan LD, Wang QS, Li XQ, Feng YM. Cancer-associated fibroblasts induce epithelialmesenchymal transition of breast cancer cells through paracrine TGF-beta signalling. Br J Cancer. 2014; 110: 724-32.

13. Tse JC, Kalluri R. Mechanisms of metastasis: epithelialto-mesenchymal transition and contribution of tumor microenvironment. J Cell Biochem. 2007; 101: 816-29.

14. Mani SA, Guo W, Liao MJ, Eaton EN, Ayyanan A, Zhou AY, Brooks M, Reinhard F, Zhang CC, Shipitsin M, Campbell LL, Polyak K, Brisken C, et al. The epithelialmesenchymal transition generates cells with properties of stem cells. Cell. 2008; 133: 704-15.

15. Thiery JP. Epithelial-mesenchymal transitions in tumour progression. Nat Rev Cancer. 2002; 2: 442-54.

16. Battula VL, Evans KW, Hollier BG, Shi Y, Marini FC, Ayyanan A, Wang RY, Brisken C, Guerra R, Andreeff M, Mani SA. Epithelial-mesenchymal transition-derived cells exhibit multilineage differentiation potential similar to mesenchymal stem cells. Stem Cells. 2010; 28: 1435-45.

17. Zhang JF, Li G, Chan CY, Meng CL, Lin MC, Chen YC, He ML, Leung PC, Kung HF. Flavonoids of Herba Epimedii regulate osteogenesis of human mesenchymal stem cells through BMP and Wnt/beta-catenin signaling pathway. Mol Cell Endocrinol. 2010; 314: 70-4.

18. Arnold SF, Tims E, McGrath BE. Identification of bone morphogenetic proteins and their receptors in human breast cancer cell lines: importance of BMP2. Cytokine. 1999; 11: 1031-7.

19. Raida M, Clement JH, Ameri K, Han C, Leek RD, Harris AL. Expression of bone morphogenetic protein 2 in breast cancer cells inhibits hypoxic cell death. International journal of oncology 2005; 26: 1465-1470.

20. Zhang XH, Wang Q, Gerald W, Hudis CA, Norton L, Smid M, Foekens JA, Massague J. Latent bone metastasis in breast cancer tied to Src-dependent survival signals. Cancer Cell. 2009; 16: 67-78. 
21. Feng Y, Li X, Sun B, Wang Y, Zhang L, Pan X, Chen X, Wang $\mathrm{X}$, Wang J, Hao X. Evidence for a transcriptional signature of breast cancer. Breast Cancer Res Treat. 2010; 122: 65-75.

22. Rosen V. BMP2 signaling in bone development and repair. Cytokine Growth Factor Rev. 2009; 20: 475-80.

23. Ducy P, Zhang R, Geoffroy V, Ridall AL, Karsenty G. Osf2/Cbfa1: a transcriptional activator of osteoblast differentiation. Cell. 1997; 89: 747-54.

24. Tandon M, Chen Z, Pratap J. Runx2 activates PI3K/Akt signaling via $\mathrm{mTORC} 2$ regulation in invasive breast cancer cells. Breast Cancer Res. 2014; 16: R16.

25. Osterix and Runx 2 cooperate to induce expression of the Colla1 promoter. Bonekey Rep. 2013; 2: 265.

26. Zheng Q, Zhou G, Morello R, Chen Y, Garcia-Rojas X, Lee B. Type $\mathrm{X}$ collagen gene regulation by Runx 2 contributes directly to its hypertrophic chondrocyte-specific expression in vivo. J Cell Biol. 2003; 162: 833-42.

27. Selvamurugan N, Partridge NC. Constitutive expression and regulation of collagenase-3 in human breast cancer cells. Mol Cell Biol Res Commun. 2000; 3: 218-23.

28. Li XQ, Du X, Li DM, Kong PZ, Sun Y, Liu PF, Wang QS, Feng YM. ITGBL1 Is a Runx2 Transcriptional Target and Promotes Breast Cancer Bone Metastasis by Activating the TGFbeta Signaling Pathway. Cancer Res. 2015; 75: 3302-13.

29. Zhang XH, Jin X, Malladi S, Zou Y, Wen YH, Brogi E, Smid M, Foekens JA, Massague J. Selection of bone metastasis seeds by mesenchymal signals in the primary tumor stroma. Cell. 2013; 154: 1060-73.

30. Kaplan RN, Rafii S, Lyden D. Preparing the "Soil": The premetastatic niche. Cancer Research. 2006; 66: 11089-93.

31. Kang Y, Siegel PM, Shu W, Drobnjak M, Kakonen SM, Cordon-Cardo C, Guise TA, Massague J. A multigenic program mediating breast cancer metastasis to bone. Cancer Cell. 2003; 3: 537-49.

32. Mundy GR. Metastasis to bone: causes, consequences and therapeutic opportunities. Nat Rev Cancer. 2002; 2: 584-93.

33. Sun J, Li J, Li C, Yu Y. Role of bone morphogenetic protein-2 in osteogenic differentiation of mesenchymal stem cells. Mol Med Rep. 2015; 12: 4230-7.

34. Cook LM, Shay G, Araujo A, Lynch CC. Integrating new discoveries into the "vicious cycle" paradigm of prostate to bone metastases. Cancer and Metastasis Reviews. 2014; 33: 511-25.

35. Komori T. Regulation of osteoblast differentiation by Runx2. Adv Exp Med Biol. 2010; 658: 43-9.

36. Enomoto H, Shiojiri S, Hoshi K, Furuichi T, Fukuyama R, Yoshida CA, Kanatani N, Nakamura R, Mizuno A, Zanma A, Yano K, Yasuda H, Higashio K, et al. Induction of osteoclast differentiation by Runx2 through receptor activator of nuclear factor-kappa B ligand (RANKL) and osteoprotegerin regulation and partial rescue of osteoclastogenesis in Runx2(-/-) mice by RANKL transgene. Journal of Biological Chemistry. 2003; 278: 23971-7.
37. Mendoza-Villanueva D, Zeef L, Shore P. Metastatic breast cancer cells inhibit osteoblast differentiation through the Runx2/CBF beta-dependent expression of the Wnt antagonist, sclerostin. Breast Cancer Research. 2011; 13:R106.

38. Okazaki M, Takeshita S, Kawai S, Kikuno R, Tsujimura A, Kudo A, Amann E. Molecular cloning and characterization of OB-cadherin, a new member of cadherin family expressed in osteoblasts. J Biol Chem. 1994; 269: 12092-8.

39. Pishvaian MJ, Feltes CM, Thompson P, Bussemakers MJ, Schalken JA, Byers SW. Cadherin-11 is expressed in invasive breast cancer cell lines. Cancer Research. 1999; 59: 947-52.

40. Kapoor P, Saunders MM, Li Z, Zhou Z, Sheaffer N, Kunze EL, Samant RS, Welch DR, Donahue HJ. Breast cancer metastatic potential: correlation with increased heterotypic gap junctional intercellular communication between breast cancer cells and osteoblastic cells. Int J Cancer. 2004; 111: 693-7.

41. Sethi T, Rintoul RC, Moore SM, MacKinnon AC, Salter D, Choo C, Chilvers ER, Dransfield I, Donnelly SC, Strieter R, Haslett C. Extracellular matrix proteins protect small cell lung cancer cells against apoptosis: a mechanism for small cell lung cancer growth and drug resistance in vivo. Nat Med. 1999; 5: 662-8.

42. Miyamoto $H$, Murakami $T$, Tsuchida $K$, Sugino $H$, Miyake H, Tashiro S. Tumor-stroma interaction of human pancreatic cancer: acquired resistance to anticancer drugs and proliferation regulation is dependent on extracellular matrix proteins. Pancreas. 2004; 28: 38-44.

43. Banerjee C, McCabe LR, Choi JY, Hiebert SW, Stein JL, Stein GS, Lian JB. Runt homology domain proteins in osteoblast differentiation: AML3/CBFA1 is a major component of a bone-specific complex. J Cell Biochem. 1997; 66: 1-8.

44. Nagel AK, Ball LE. O-GlcNAc modification of the runtrelated transcription factor 2 (Runx2) links osteogenesis and nutrient metabolism in bone marrow mesenchymal stem cells. Mol Cell Proteomics. 2014; 13: 3381-95.

45. Rahman MS, Akhtar N, Jamil HM, Banik RS, Asaduzzaman SM. TGF-beta/BMP signaling and other molecular events: regulation of osteoblastogenesis and bone formation. Bone Res. 2015; 3: 15005.

46. Mesilaty-Gross S, Anikster Y, Vilensky B, Wolf I, Phillip M, Gat-Yablonski G. Different patterns of human serum procollagen C-proteinase enhancer1 (PCPE1). Clin Chim Acta. 2009; 403: 76-80.

47. Sasaki H, Yu CY, Dai M, Tam C, Loda M, Auclair D, Chen LB, Elias A. Elevated serum periostin levels in patients with bone metastases from breast but not lung cancer. Breast Cancer Res Treat. 2003; 77: 245-52.

48. Le Gall C, Bellahcene A, Bonnelye E, Gasser JA, Castronovo V, Green J, Zimmermann J, Clezardin P. A cathepsin K inhibitor reduces breast cancer induced osteolysis and skeletal tumor burden. Cancer Res. 2007; 67: 9894-902. 
49. Stein GS, Lian JB, van Wijnen AJ, Stein JL, Montecino M, Javed A, Zaidi SK, Young DW, Choi JY, Pockwinse SM. Runx 2 control of organization, assembly and activity of the regulatory machinery for skeletal gene expression. Oncogene. 2004; 23: 4315-29.

50. Quarto N, Li S, Renda A, Longaker MT. Exogenous activation of BMP-2 signaling overcomes TGFbetamediated inhibition of osteogenesis in Marfan embryonic stem cells and Marfan patient-specific induced pluripotent stem cells. Stem Cells. 2012; 30: 2709-19.
51. Sato R, Semba T, Saya H, Arima Y. Concise Review: Stem Cells and Epithelial-Mesenchymal Transition in Cancer: Biological Implications and Therapeutic Targets. Stem cells 2016; 34: 1997-2007.

52. Du X, Li XQ, Li L, Xu YY, Feng YM. The detection of ESR1/PGR/ERBB2 mRNA levels by RT-QPCR: a better approach for subtyping breast cancer and predicting prognosis. Breast Cancer Res Treat. 2013; 138: 59-67. 